\title{
Formation and propagation of cosmic-ray ensembles
}

\section{Oleksandr Sushchov ${ }^{a, *}$ on behalf of the CREDO Collaboration}

(a complete list of authors can be found at the end of the proceedings)

${ }^{a}$ Institute of Nuclear Physics Polish Academy of Sciences, Radzikowskiego 152, 31-342 Kraków, Poland

E-mail: oleksandr.sushchov@ifj.edu.pl

High-energy particles undergo different interactions while propagating through the Universe. As a result, they initiate particle cascades of various types and sizes, referred to as cosmic-ray ensembles (CRE). Since recently, Cosmic-Ray Extremely Distributed Observatory (CREDO) Collaboration aims at pursuing a mission dedicated to CRE, since this observation channel, i.e. correlated observation of cosmic rays on the global scale, complements the current approach to cosmic-ray research, which focuses on air showers initiated by individual cosmic rays. Recent results of Monte Carlo simulations showing that there might be a chance of observing a CRE originating from synchrotron radiation occurring even as far away from the Earth as at distances exceeding the Galaxy size, are presented. The issues and perspectives of the CRE-oriented research are discussed as well.

$37^{\text {th }}$ International Cosmic Ray Conference (ICRC 2021)

July 12th - 23rd, 2021

Online - Berlin, Germany

\footnotetext{
${ }^{*}$ Presenter
} 


\section{Introduction}

The origin of ultra-high energy cosmic rays (UHECR; $E>10^{18} \mathrm{eV}$ ) still poses a challenge for astrophysics. Theoretical models proposed for its explanation can be divided into two groups. One approach (bottom-up) studies particle acceleration up to ultra-high energies (UHE) [1], with the shock wave structures in active galactic nuclei (AGN), gamma-ray bursts (GRB), jets of radio galaxies etc. considered as the most likely accelerator sites. Another group of scenarios (top-down) anticipates UHECR origination resulting from decay or annihilation of supermassive particles [2]. The existence of UHE photons is not excluded by either approach, though estimations of their fraction in the UHECR flux are different: from percents in the bottom-up models [3] to around a half in the top-down models [4]. New physics scenarios such as Lorentz invariance violation (LIV) [5] can also be tested with UHE photons, since the increase of the photons mean free path, which leads to extension of the photon flux, is one of LIV consequences. However, none of UHE photons have been unambiguously identified by the main cosmic ray experiments so far, setting upper limits restricting under some assumptions top-down scenarios [6, 7]. Still, the search for UHE photons remains among tempting and ambitious tasks. Moreover, the physics of the UHE regime could be not understood properly, since it is described by the interaction models relying on the extrapolated lower energies data. Thus, the exact interpretation of the UHECR data might be complicated due to the growth of uncertainties in the UHE range. Thereby, potential observations of UHE photons are extremely important for many astrophysical problems.

However, uncertainties in the UHECR data treatment leave some room for alternative theories about UHE photons existence. For instance, a possible explanation of their non-observation could be related to their negligible chances to reach the Earth because of the interactions during their propagation. Indeed, high energy particles, including photons, should interact with matter, fields and different types of radiation while propagating in space. These interactions obviously generate particle cascades of various constituents and spatial extent, hereafter referred to as cosmic-ray ensembles (CRE). The state-of-the-art cosmic ray experiments rely on the detection and analysis of cosmic rays with individual detectors or arrays, but the study of cosmic particles correlated on the global scale in space and time shows slower progress. Several years ago Cosmic-Ray Extremely Distributed Observatory (CREDO) Collaboration [8] initiated a research program dedicated to the study of CRE, since an expected output from this investigation could help answering many yet puzzling astrophysical questions related to the highest known energy domain.

We present a method of investigation of the processes taking place during the propagation of high energy particles through the Universe. We are particularly interested in UHE electrons, either accelerated in certain astrophysical conditions or generated in pair production process by UHE photons, which are believed to initiate the largest CRE. We study the circumstances under which it is potentially possible to expect correlated arrival of at least two cosmic rays belonging to one CRE at the Earth. 


\section{Simulations}

\subsection{CRPropa 3}

The basic software chosen for the simulation of propagation of cosmic ray particles leading to the formation of CRE is CRPropa 3 [9]. It is one of the most popular state of the art Monte Carlo codes which allows to simulate behavior of different particles (leptons, nuclei, photons etc.) in astrophysical environments, accounting for their interactions with background radiation, fields and matter as well as cosmological effects. It is designed in accordance with the standards for modular codes. Each of the important simulation aspects (sources, observers, interactions, magnetic field, breaking conditions, etc.) is programmed as a separate module, independent from the others. The user can modify already existing modules or add his own (written either in $\mathrm{C}++$ or in Python), using any combination and consequence of them according to his task.

All this makes CRPropa 3 a very helpful and flexible instrument for solving a wide range of high-energy astrophysics problems. Its official website [10] provides the user with the updates, usage examples and tutorials.

\subsection{CRE-Pro script}

Our present research is dedicated to studying the propagation of charged particles in the electromagnetic fields leading to synchrotron emission of photons, which are the main constituents of an electromagnetic particle cascade, such as a CRE. Synchrotron photons, massively generated by CRPropa as a result of the energy loss of a parent electron in the magnetic field, propagate linearly. Since their number is large, tracking them within the CRPropa simulation chain consumes a great amount of computational resources, often causing memory problems. We developed an algorithm, later referred as the "CRE-Pro" script, which allows to replace tracking of photons by CRPropa with the estimation of the CRE footprint size directly from simple geometrical considerations.

The easiest and most reliable way of defining the trajectory of a particle is the use of iterative methods in order to solve differential equations of motion in an environment, permeated by magnetic field, which affects the particle propagation. The trajectory of a particle could be treated as a polygonal chain consisting of a series of line segments (propagation steps).

The main idea behind the CRE-Pro is that all synchrotron photons emitted along the propagation step $A C$ (Fig. 1) due to their linear propagation are confined within some area $A_{1} C_{1}$ of the observer surface. This area is in a sense the "projection" of the step onto the surface, while its length $D_{C R E}$ is the size of the CRE formed by the photons emitted along the given step.

Let the electron of energy $E$ propagate in the magnetic field which transverse component is $B$. The gyroradius of the electron is then

$$
R_{g}=\frac{\gamma m v_{\perp}}{B|q|}=\frac{E}{e c B}
$$

thus a linear propagation step with the length $D_{\text {step }}$ is an approximation of an $\operatorname{arc} A C$ of the angular size $\alpha$, where

$$
\alpha=\frac{D_{\text {step }}}{R_{g}}
$$




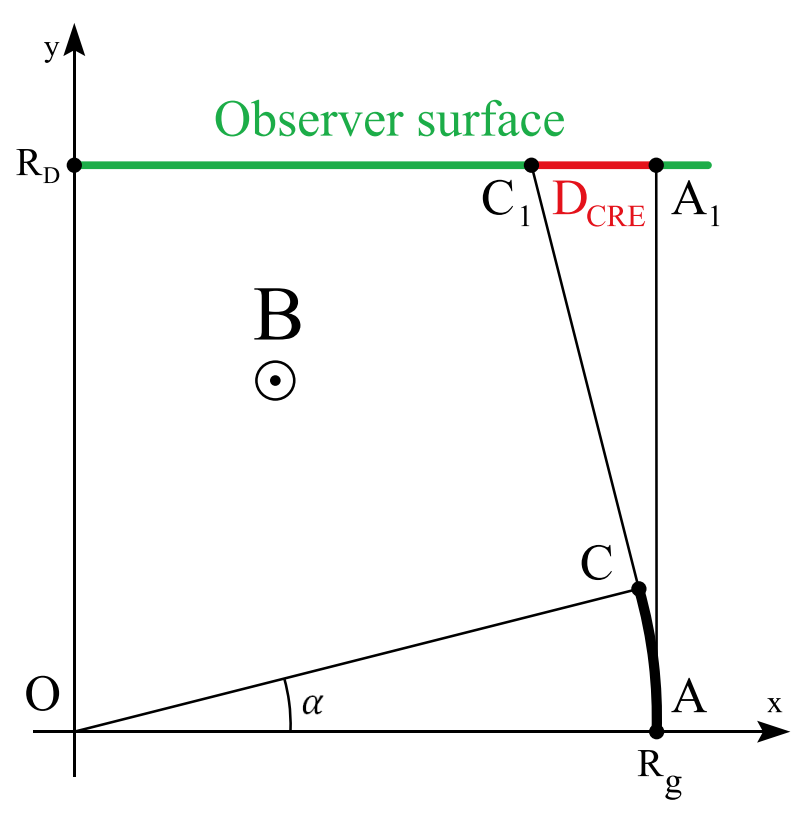

Figure 1: Geometrical interpretation of the CRE-Pro algorithm

The photons generated along a given step should propagate along the lines tangent to the arc at the emission points, thus such lines at the beginning and at the end of the step define the edges of the step projection onto the observer surface. The latter was chosen in the simplest way, as a plane located at a distance $R_{D}$ from the start of the step $A$ perpendicularly to the tangent line at this point. As one can easily see, $D_{C R E}$ as a function of $\alpha, B, R_{D}$ and $E$ can be expressed as

$$
D_{C R E}=A_{1} C_{1}=R_{g}-\frac{R_{g}}{\cos \alpha}+R_{D} \tan \alpha .
$$

The strength of the magnetic field $B$ and electron energy $E$ are read by the CRE-Pro script from the CRPropa output. Both these values, generally speaking, change along the step, but to assure that the maximum CRE size $D_{C R E}$ is calculated, we use the energy at the end of the step (which is obviously smaller than at its beginning), and the maximal value of magnetic field along the step.

Since we defined a CRE as at least 2 correlated particles arriving to the observer, the exact distribution of the photons along the CRE footprint is important when defining the maximal observer distance such a CRE could originate at. From the CRPropa output only the photons' emission points are known (their propagation is replaced with CRE-Pro), thus we modelled the distribution of photons along the footprint in two different ways. The simplest case is the equidistant photons distribution. To expect 2-photon CRE at the Earth sized area, one should insert the condition $(N-1) D_{\text {Earth }}=D_{C R E}$, where $N$ is the number of photons emitted along the step, into Eq. 3 , to obtain the maximum observer distance for the 2-photon CRE not exceeding the Earth size in a form: 


$$
R_{D(2)}=\frac{(N-1) D_{E a r t h} \cos \alpha+R_{g}(1-\cos \alpha)}{\sin \alpha} .
$$

Another approach we used to account for the distribution of photons along the step projection on the observer plane is keeping proportionality of the corresponding distances between the emission points of the photons and their projections on the observer plane. In this case

$$
\frac{D_{\text {step }}}{D_{i, i+1}} D_{\text {Earth }}=D_{C R E},
$$

where $D_{i, i+1}$ is the distance between the $\mathrm{i}$-th and the (i+1)-th photons, and, finally, the sought observer distance for the 2-photon CRE is

$$
R_{D(2)}=\frac{\left(D_{\text {step }} / D_{i, i+1}\right) D_{\text {Earth }} \cos \alpha+R_{g}(1-\cos \alpha)}{\sin \alpha}
$$

It should be noted that the "equidistant" approach is completely artificial; in a sense, it describes the conditions for the CRE consisting of an "average" pair of photons. On the other hand, the "proportional" case, might also not reflect the real distribution of photons properly, but it is more realistic than the "equidistant" one.

\section{Results}

We summarize our results from the CRPropa simulations of synchrotron emission of UHE electrons propagating in the galactic magnetic field followed by the post-processing of the simulation output using CRE-Pro script. Two astrophysical models of UHE electrons distribution have been analyzed this way.

\subsection{Galactic Center as a UHECR source}

Within the first of the considered scenarios it is assumed that the super-massive black hole in the Galactic Center (GC) could be a source of such electrons, since violent processes are expected to take place in this object and its vicinity (see, e.g. [11] and references therein).

We chose 11 random directions of the initial momentum of the electron, and varied its starting energy from $10^{17} \mathrm{eV}$ to $10^{19} \mathrm{eV}$ with a logarithmic step size 0.1 , thus using in the simulations 21 values of the electron starting energy overall. Each run of the electron with a specific starting energy in a certain direction was repeated 10 times which resulted in 2310 simulation runs in total.

For every step of a simulation run we have calculated the observer distance for a 2-photon CRE, $R_{D(2)}$, for equidistant distribution of photons along the CRE footprint, using Eq. 4 . For the proportional case $R_{D(2)}$ was computed for every neighboring photons pair (Eq. 6), after which the maximal, "most optimistic", value was selected. These values were plotted in Fig. 2 to compare two cases of the photons distribution, accounting for every propagation step of each of 10 runs in a given direction with a certain starting energy (Fig. 2a), and, correspondingly, all the steps of 210 runs in a given direction, regardless of the starting energy of the electron (Fig. 2b). As one can see, the equidistant photon distribution allows for making qualitative conclusions: the average photon pairs are not expected to form CRE observable on Earth, unless the sources (interaction origins) are very close, like the nearest stars. Proportional model gives a lot more optimistic expectations 


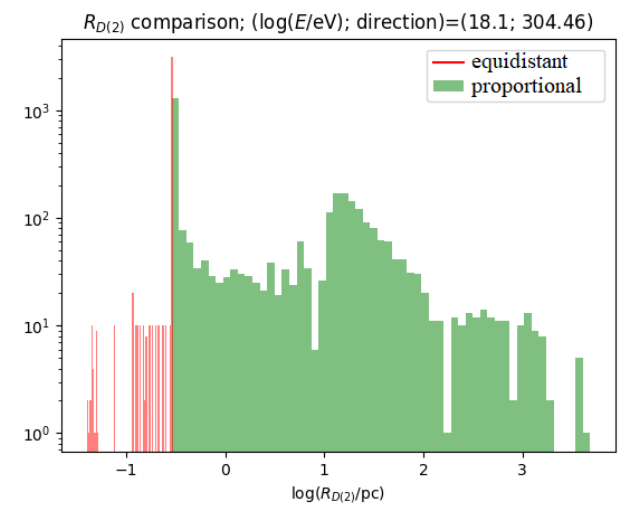

(a) 3310 entries; the largest "equidistant" $R_{D(2)}$ is $0.296 \mathrm{pc}$, the largest "proportional" $R_{D(2)}$ is $4.863 \mathrm{kpc}$

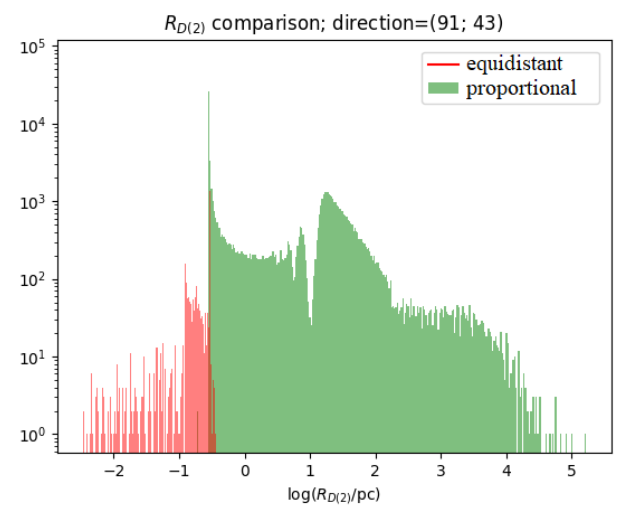

(b) 81751 entries; the largest "equidistant" $R_{D(2)}$ is $0.383 \mathrm{pc}$, the largest "proportional" $R_{D(2)}$ is $167.280 \mathrm{kpc}$

Figure 2: Comparison of two approaches to the distribution of photons over the observer surface for the GC model

of the distances between the emission region and the observer. There might be a chance to observe a CRE of the Earth size originated at distances exceeding the Galaxy size. This conclusion holds true for all the directions chosen for this set of simulations, as well as for every value of the electron starting energy from the considered range.

\subsection{Uniform distribution of galactic sources}

Another theory attempting to explain origination and existence of UHECR, including UHE electrons, is super heavy dark matter (SHDM) scenario. According to it, super massive X particles (see, e.g., [12], [13], [14]) may decay or annihilate, producing quarks and leptons. Quarks then hadronize (produce jets of hadrons consisting mostly of pions with a small fraction of nucleons). Consecutive pion decays result in photons, neutrinos and electrons production. Within this scenario neither acceleration mechanisms nor distinct astrophysical sources are required. Since it is believed that most of the mass in galactic halos is composed of dark matter, described interactions may occur elsewhere in the Galaxy. Respectively, SHDM model assumes uniform distribution of particles within the halo or even within a cluster of galaxies [15].

We simulated uniform distribution of points over the Galaxy, considering the latter as the sphere of $20 \mathrm{kpc}$ radius. 292 random points were selected as the origin points of $1 \mathrm{EeV}$ electrons, each being initially directed towards the Solar System. Every propagation step of each run was post-processed with CRE-Pro as described above. As a result, we compare the calculations of $R_{D(2)}$ for two models of the photons distribution over the observer surface in the cumulative plot (Fig. 3). The equidistant distribution allows for making the same qualitative conclusions as in the previous scenario, while for the proportional distribution one can indicate numerous entries corresponding to the observer distances exceeding the Galaxy size. 


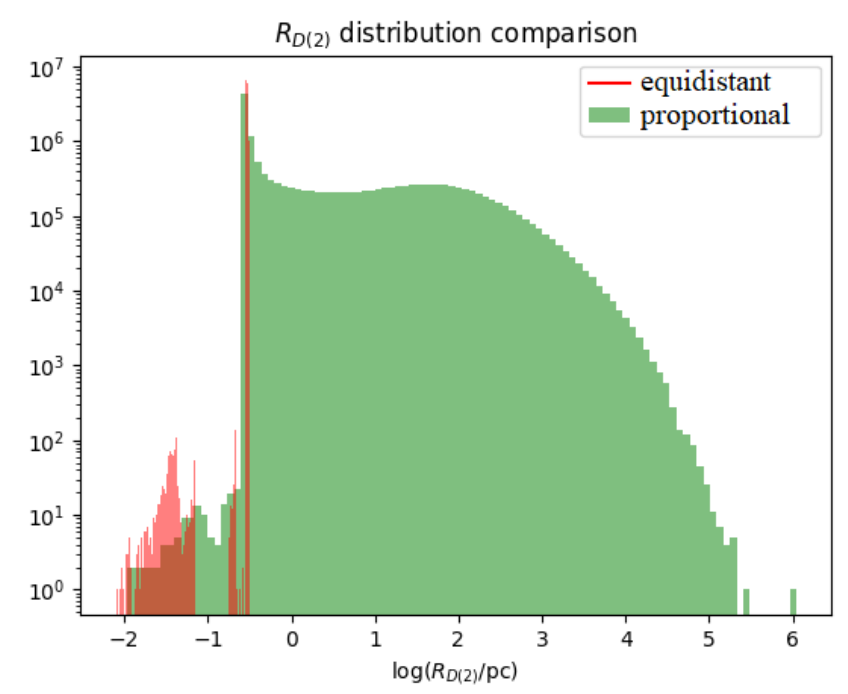

Figure 3: Comparison of two approaches to the distribution of photons over the observer surface for the SHDM model. 15318555 entries, the largest value of equidistant $R_{D(2)}$ is $0.321 \mathrm{pc}$, the largest value of the proportional $R_{D(2)}$ is $1.129 \mathrm{Mpc}$.

\section{Summary}

If UHE electrons propagate through the Universe, they are subjected to several processes. The most inevitable of them is energy loss via synchrotron emission, since magnetic fields permeate the Cosmos. We have performed simulations of synchrotron emission by UHE electrons during their propagation in the GMF, considering two toy models of distribution of their sources. The results are compared in Fig. (4), which is a normalized histogram of the maximum observer distances for a 2-photon CRE $R_{D(2)}$, calculated for the proportional distribution of photons along the observer surface for every step of the primary electron propagation. The initial energy of primary electrons is $1 \mathrm{EeV}$ for both scenarios.

As one can see from the plot, CRE of two photons separated no more than the Earth diameter have chances to be formed at distances comparable with the Galactic size ( tens of kpc in the Galactic center source model) or even exceeding it ( $\sim 1 \mathrm{Mpc}$ in case of uniform distribution of the sources over the Galaxy). The latter case might allow for some extragalactic sources to initiate the observable CRE, but the study of extragalactic physics conditions in the CRE context requires additional research.

\section{Acknowledgments}

Numerical simulations have been performed using computational and storage resources of the Prometheus system at ACC Cyfronet AGH and we warmly thank the staff for their always helpful supercomputing support. 


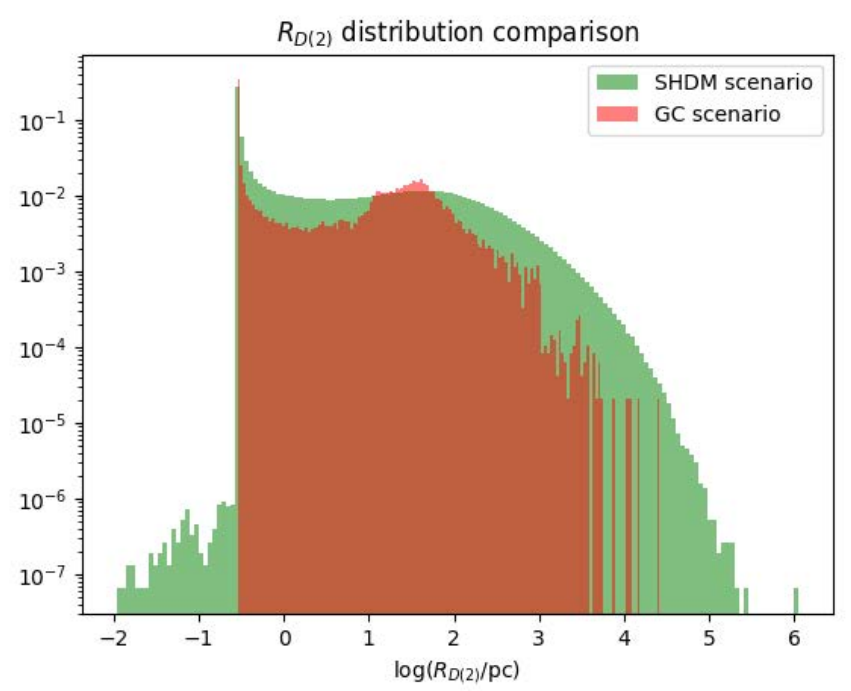

Figure 4: Comparison of two scenarios of UHECR sources distribution. The number of entries for the "SHDM" scenario is 15318555 , the largest value of the proportional $R_{D(2)}$ is $1.129 \mathrm{Mpc}$. For the "Galactic center" scenario the plot contains 48339 entries, with the largest $R_{D(2)}$ value $26.146 \mathrm{kpc}$.

\section{References}

[1] M. S. Longair. High Energy Astrophysics. Feb. 2011.

[2] V. A. Kuzmin and V. A. Rubakov, Phys. Atom. Nucl. 61, 1028.

[3] G. B. Gelmini, O. E. Kalashev, D. V. Semikoz. JETP, 106, 1061.

[4] V. Berezinsky, M. Kachelrieß, and A. Vilenkin. Phys. Rev. Lett. 79, 4302.

[5] L. Maccione, S. Liberati, G. Sigl. Phys. Rev. Lett. 105, 021101.

[6] T. Abu-Zayyad et al. (Telescope Array Collaboration). Phys. Rev. D 88, 112005.

[7] A. Aab et al. JCAP09(2020)E02.

[8] P. Homola et. al. Symmetry 2020, 12(11), 1835.

[9] R. Alves Batista, et al. JCAP05(2016)038.

[10] https://crpropa.desy.de/.

[11] A. Tursunov, et al. 2020 ApJ 89799.

[12] M. Birkel and S. Sarkar. Astropart.Phys.9:297-309,199.

[13] P. H. Frampton, B. Keszthelyi, Y.J. Ng. Int.J.Mod.Phys.D 8 (1999) 117-122.

[14] P. Blasi, R. Dick, E. W. Kolb. Astropart.Phys. 18 (2002) 57-66.

[15] P. Bhattacharjee and G. Sigl. 2000 Physics Reports 327.3109. 


\section{Full Authors List: CREDO Collaboration}

Oleksandr Sushchov $^{1}$, Piotr Homola ${ }^{1}$, David E. Alvarez Castillo ${ }^{1,2}$, Dmitriy Beznosko ${ }^{3}$, Nikolai Budnev ${ }^{4}$, Dariusz Góra ${ }^{1}$, Alok C. Gupta $^{5}$, Bohdan Hnatyk ${ }^{6}$, Marcin Kasztelan ${ }^{7}$, Peter Kovacs ${ }^{8}$, Bartosz Łozowski ${ }^{9}$, Mikhail V. Medvedev ${ }^{10,11}$, Justyna Miszczyk ${ }^{1}$, Alona Mozgova $^{6}$, Vahab Nazari ${ }^{2}, 1$, Michal Niedźwiecki ${ }^{12}$, Maciej Pawlik ${ }^{13,14}$, Matías Rosas ${ }^{15}$, Krzysztof Rzecki ${ }^{14}$, Katarzyna Smelcerz ${ }^{12}$, Karel Smolek ${ }^{16}$, Jaroslaw Stasielak ${ }^{1}$, Slawomir Stuglik ${ }^{1}$, Manana Svanidze ${ }^{17}$, Arman Tursunov ${ }^{18}$, Yuri Verbetsky ${ }^{17}$, Tadeusz Wibig ${ }^{19}$, Jilberto Zamora-Saa ${ }^{20}$.

${ }^{1}$ Institute of Nuclear Physics Polish Academy of Sciences, Radzikowskiego 152, 31-342 Kraków, Poland.

${ }^{2}$ Joint Institute for Nuclear Research, Dubna, 141980 Russia.

${ }^{3}$ Clayton State University, Morrow, Georgia, USA.

${ }^{4}$ Irkutsk State University, Russia.

${ }^{5}$ Aryabhatta Research Institue of Observational Sciences (ARIES), Manora Peak, Nainital 263001, India.

${ }^{6}$ Astronomical Observatory of Taras Shevchenko National University of Kyiv, 04053 Kyiv, Ukraine.

${ }^{7}$ National Centre for Nuclear Research, Andrzeja Soltana 7, 05-400 Otwock-Świerk, Poland.

${ }^{8}$ Institute for Particle and Nuclear Physics, Wigner Research Centre for Physics, 1121 Budapest, Konkoly-Thege Miklós út 29-33, Hungary

${ }^{9}$ Faculty of Natural Sciences, University of Silesia in Katowice, Bankowa 9, 40-007 Katowice, Poland.

${ }^{10}$ Department of Physics and Astronomy, University of Kansas, Lawrence, KS 66045, USA.

${ }^{11}$ Laboratory for Nuclear Science, Massachusetts Institute of Technology, Cambridge, MA 02139, USA.

${ }^{12}$ Department of Computer Science, Faculty of Computer Science and Telecommunications, Cracow University of Technology, Warszawska 24, 31-155 Kraków, Poland.

${ }^{13}$ ACC Cyfronet AGH-UST, 30-950 Kraków, Poland.

${ }^{14}$ AGH University of Science and Technology, Mickiewicz Ave., 30-059 Kraków, Poland.

${ }^{15}$ Liceo 6 Francisco Bauzá, Montevideo, Uruguay.

${ }^{16}$ Institute of Experimental and Applied Physics, Czech Technical University in Prague.

${ }^{17}$ E. Andronikashvili Institute of Physics under Tbilisi State University, Georgia.

${ }^{18}$ Research Centre for Theoretical Physics and Astrophysics, Institute of Physics, Silesian University in Opava, Bezručovo nám. 13, CZ-74601 Opava, Czech Republic.

${ }^{19}$ University of Łódź, Faculty of Physics and Applied Informatics, 90-236 Łódź, Pomorska 149/153, Poland.

${ }^{20}$ Universidad Andres Bello, Departamento de Ciencias Fisicas, Facultad de Ciencias Exactas, Avenida Republica 498, Santiago, Chile. 